\title{
Chemical abundances of planetary nebulae towards the Galactic anticenter ${ }^{\star} \star \star$
}

\author{
R. D. D. Costa, M. M. M. Uchida, and W. J. Maciel
}

\author{
Instituto de Astronomia, Geofísica e Ciências Atmosféricas (IAG), Universidade de São Paulo, Rua do Matão 1226, \\ 05508-900, São Paulo SP, Brazil \\ e-mail: [roberto;monica;maciel]@astro.iag.usp.br
}

Received 20 October 2003 / Accepted 24 April 2004

\begin{abstract}
In this paper we report new observations and derive chemical abundances for a sample of 26 planetary nebulae (PN) located in the anticenter direction. Most of these nebulae are far away objects, located at Galactocentric distances greater than about $8 \mathrm{kpc}$, so that they are particularly useful for the determination of the radial gradients at large distances from the galactic center. A comparison of the present results with previously determined abundances suggests that the radial abundance gradients flatten out at distances larger than about $10 \mathrm{kpc}$ from the center.
\end{abstract}

Key words. ISM: planetary nebulae: general - Galaxy: disk - ISM: abundances

\section{Introduction}

The presence of radial abundance gradients for several element ratios in the Milky Way is now well established (see Maciel 1997, 2000, for a revision). Gradients can be derived from different kinds of objects, such as O, B stars, HII regions or planetary nebulae, and seem to be a common property of spiral galaxies. In fact, there are reports of gradients in many other galaxies apart from the Milky Way from the analysis of HII regions (Searle 1971; Smith 1975; Blair et al. 1982; Kennicutt \& Garnett 1996; Ferguson et al. 1998).

The study of radial gradients involves one of the most important constraints for galactic evolution models, as they can provide at least three crucial information items: i) the magnitude of the gradient itself, generally measured in dex/kpc; ii) possible variations in the gradient for different galactocentric distances; iii) the time variation in the gradients.

Regarding the space variations of the gradients, presently available results are somewhat controversial. Some results for the Milky Way based on stars (Kaufer et al. 1994) and HII regions (Esteban \& Peimbert 1995; Vílchez \& Esteban 1996) suggest decreasing gradients for galactocentric distances $R>$ $6 \mathrm{kpc}$. This is confirmed by a recent analysis based on planetary nebulae (Maciel \& Quireza 1999), and is also supported by chemical evolution models by Chiappini et al. (1997) and

^ Based on observations made at the European Southern Observatory (Chile) and Laboratório Nacional de Astrofísica (Brazil).

$\star \star$ The full Table 2 is only available in electronic form at the CDS via anonymous ftp to cdsarc.u-strasbg.fr $(130.79 .128 .5)$ or via http://cdsweb.u-strasbg.fr/cgi-bin/qcat?J/A+A/423/199
Matteucci (2000). However, no significant variations in the gradient are detected from a sample of O, B stars by Smartt (2000, and references therein), and a flattened gradient near the solar neighbourhood has been suggested by Andrievsky et al. (2002, and references therein) on the basis of a sample of Cepheid variables.

The shapes of the gradients in spiral galaxies are also subject to discussion. Some flattening in the outer regions has been proposed (Díaz 1989), while some recent results are consistent with an exponential gradient with a constant slope (Henry \& Howard 1995).

Planetary nebulae can play an important role in this context (Maciel \& Köppen 1994; Peimbert \& Carigi 1998), as nebular abundances can be accurately derived, even for objects at large heliocentric distances, which is particularly useful for objects in the direction of the galactic anticenter (Maciel \& Quireza 1999). Also, these objects can be classified according to the masses and ages of their progenitors, so that they are also useful in the study of the time variations of the gradients (Maciel \& Costa 2003; Maciel et al. 2003). The derivation of chemical abundances for a homogeneous and wide sample of PN at large galactocentric distances, located basically outside the solar circle of about $8 \mathrm{kpc}$, would therefore provide the tools for the assessment of the gradient properties at these large distances.

In this work, we report the chemical abundances for a sample of PN towards the galactic anticenter, where most of the objects placed at large galactocentric distances are located. They were selected from the ESO-Strasbourg Galactic PN Catalog (Acker et al. 1992), and the sample consists of 26 objects, all of them observed, reduced and analysed homogeneously. 
Table 1. Log of the observations and distances.

\begin{tabular}{lccclcr}
\hline \hline Object & PN G & RA & Dec & Place \& date & $d$ & $R$ \\
\hline A 12 & $198.6-06.3$ & 060223 & 093903 & ESO - Dec. 1999 & 2.0 & 9.5 \\
A 18 & $216.0-00.2$ & 065615 & -025312 & ESO - Dec. 1999 & 1.6 & 8.9 \\
A 20 & $214.9+07.8$ & 072301 & 014537 & ESO - Dec. 2000 & 2.0 & 9.3 \\
H 3-75 & $193.6-09.5$ & 054044 & 122116 & ESO - Dec. 1999 & 2.4 & 9.9 \\
IC 2003 & $161.2-14.8$ & 035622 & 335230 & LNA - Dec. 1995 & 4.7 & 12.0 \\
IC 2165 & $221.3-12.3$ & 062143 & -125910 & LNA - Dec. 1995 & 2.0 & 9.2 \\
J 320 & $190.3-17.7$ & 050534 & 104221 & LNA - Dec. 1995 & 6.1 & 13.4 \\
J 900 & $194.2+02.5$ & 062557 & 174727 & LNA - Dec. 1995 & 2.8 & 10.3 \\
K 1-7 & $197.2-14.2$ & 053148 & 065609 & LNA - Dec. 1995 & 3.8 & 11.2 \\
K 2-1 & $173.7-05.8$ & 050309 & 305003 & ESO - Dec. 2000 & 1.1 & 8.7 \\
K 3-66 & $167.4-09.1$ & 043637 & 333930 & LNA - Dec. 1995 & 6.7 & 14.1 \\
K 3-70 & $184.6+00.6$ & 055845 & 251844 & ESO - Dec. 1999 & & \\
M 1-6 & $211.2-03.5$ & 063545 & -000541 & ESO - Dec. 1999 & 2.6 & 9.9 \\
M 1-7 & $189.8+07.7$ & 063721 & 240030 & ESO - Dec. 1999 & 5.9 & 13.4 \\
M 1-8 & $210.3+01.9$ & 065334 & 030824 & LNA - Dec. 1995 & 3.4 & 10.7 \\
M 1-9 & $212.0+04.3$ & 070519 & 024659 & LNA - Dec. 1995 & 4.9 & 12.0 \\
M 1-13 & $232.4-01.8$ & 072115 & -180836 & LNA - Dec. 1995 & 5.3 & 11.6 \\
M 1-14 & $234.9-01.4$ & 072756 & -201323 & LNA - Dec. 1995 & 4.0 & 10.4 \\
M 1-16 & $226.7+05.6$ & 073719 & -093848 & LNA - Dec. 1995 & 5.5 & 12.0 \\
M 1-17 & $228.8+05.3$ & 074022 & -113230 & LNA - Dec. 1995 & 7.4 & 13.6 \\
M 1-18 & $231.4+04.3$ & 074206 & -142141 & ESO - Dec. 1999 & 4.4 & 10.9 \\
M 3-2 & $240.3-07.6$ & 071450 & -275020 & ESO - Dec. 1999 & 8.8 & 14.1 \\
M 3-3 & $221.7+05.3$ & 072635 & -052157 & ESO - Dec. 1999 & 5.7 & 12.4 \\
NGC 1514 & $165.5-15.2$ & 040922 & 304600 & ESO - Dec. 2000 & 0.8 & 8.3 \\
SA 2-21 & $238.9+07.3$ & 080843 & -191359 & ESO - Dec. 1999 & 3.3 & 9.7 \\
YC 2-5 & $240.3+07.0$ & 081042 & -203133 & ESO - Dec. 1999 & 7.7 & 13.2 \\
\hline
\end{tabular}

Table 2. Line fluxes (available electronically at the CDS).

\begin{tabular}{lrrrrrrrr}
\hline \hline Ion & A 12 & A 18 & A 20 & H3-75 & IC 2003 & IC 2165 & J 320 & J 900 \\
\hline H $\gamma 4340$ & 54.0 & 34.0 & 27.0 & 68.0 & 26.0 & 53.0 & 63.0 & 59.0 \\
[O III] 4363 & 8.7 & 10.6 & 3.8 & 13.4 & 11.5 & 14.3 & 13.6 & 9.6 \\
$\cdots$ & $\ldots$ & $\ldots$ & $\ldots$ & $\cdots$ & $\cdots$ & $\ldots$ & $\cdots$ & $\cdots$ \\
\hline
\end{tabular}

\section{Observations}

The observations were carried out at two different observatories: at ESO/La Silla, and at LNA/Brazil. At ESO we used the $1.52 \mathrm{~m}$ telescope and the Boller \& Chivens Cassegrain spectrograph, in two observational runs: Dec./1999 and Dec./2000. We used grating \#23 and CCD \#38, which provide a dispersion of $2.2 \AA /$ pix and spectral coverage of about $5000 \AA$. The effective wavelength coverage was reduced to about $3500 \AA$ starting at $4200 \AA$, due to a low $\mathrm{S} / \mathrm{N}$ ratio shortward of this wavelength. At LNA, in Dec./1995, we used the $1.60 \mathrm{~m}$ telescope, a Boller \& Chivens Cassegrain spectrograph and a combination grating $+\mathrm{CCD}$, which gives a dispersion of $4.5 \AA /$ pixel and spectral coverage of about $4500 \AA$. An east-west long slit of 2 arcsecs width was used for all obervations. Table 1 gives the logbook of the observations and also the adopted distances $d(\mathrm{kpc})$ to the
Sun and galactocentric distances $R(\mathrm{kpc})$, which we will use in Sect. 4. Flux calibration was secured through the observation of at least three spectrophotometric standards in each night. Each object was observed at least twice, and line fluxes were derived from the average of all good measurements for each object.

Data reduction was performed using the IRAF package, and followed the standard procedure for long slit spectroscopy: correction of bias, dark and flat-field, extraction of the spectral profile, wavelength calibration and flux calibration. Atmospheric extinction was corrected through the mean coefficients for the observatory and season. Line fluxes were calculated assuming Gaussian profiles, and a Gaussian deblending routine was used when necessary. Fluxes calculated for all diagnostic lines are listed in Table 2. Interstellar reddening was derived from the difference between measured and predicted values for the Balmer ratio $\mathrm{H} \alpha / \mathrm{H} \beta$, assuming 
Table 3. Physical parameters.

\begin{tabular}{lrrrr}
\hline \hline Object & $E(B-V)$ & $T_{\mathrm{e}}[\mathrm{OIII}]$ & $T_{\mathrm{e}}[\mathrm{NII}]$ & $n_{\mathrm{e}}[\mathrm{SII}]$ \\
\hline A 12 & 0.440 & 11912 & 9144 & 255 \\
A 18 & 0.969 & 15736 & 14317 & 364 \\
A 20 & 0.0 & & & 5000 \\
H 3-75 & 0.290 & 12813 & 9013 & 119 \\
IC 2003 & 0.301 & 12080 & 13276 & 5144 \\
IC 2165 & 0.417 & 12519 & 14075 & 5715 \\
J 320 & 0.248 & 11944 & 12815 & 4915 \\
J 900 & 0.558 & 11320 & 12513 & 4016 \\
K 1-7 & 0.144 & 16123 & 11329 & 92 \\
K 2-1 & 0.433 & 11375 & & 493 \\
K 3-66 & 0.660 & 9057 & 13143 & 4725 \\
K 3-70 & 1.530 & 17500 & 11643 & 2361 \\
M 1-6 & 1.260 & 10558 & 11306 & 8426 \\
M 1-7 & 0.129 & 10244 & 11016 & 1135 \\
M 1-8 & 0.374 & 15563 & 10307 & 482 \\
M 1-9 & 0.540 & 10922 & 10435 & 4516 \\
M 1-13 & 0.208 & 8196 & 9601 & 994 \\
M 1-14 & 0.757 & 12805 & 9987 & 6784 \\
M 1-16 & 0.450 & 10657 & 10351 & 2311 \\
M 1-17 & 0.650 & 10008 & 10525 & 6030 \\
M 1-18 & 0.088 & 11224 & 10896 & 69 \\
M 3-2 & 0.317 & & 10164 & 230 \\
M 3-3 & 0.250 & 13826 & 9867 & 349 \\
NGC 1514 & 0.410 & & & 5000 \\
SA 2-21 & 0.200 & 12720 & 15152 & 351 \\
YC 2-5 & 0.290 & 11477 & 11477 & 5000 \\
\hline
\end{tabular}

Case B (Osterbrock 1989), and adopting the interstellar reddening law by Cardelli et al. (1989), with $R_{V}=3.1$. The derived $E(B-V)$ values are given in Table 3 for each nebula.

A preliminary analysis of the objects observed in 1995 has already been published (Costa et al. 1997); however, for the present work we rederived the physical parameters and abundances for the whole sample, in order to assure the homogeneity of the results.

\section{Physical parameters and abundances}

Electron densities $n_{\mathrm{e}}$ were derived from the [SII] $\lambda 6717 / 6731$ ratio, while electron temperatures $T_{\mathrm{e}}$ were estimated from both the [OIII] $\lambda 4364 / 5007$ and $[\mathrm{NII}] \lambda 5755 / 6584$ line ratios. When both line ratios were available, the [OIII] temperature was used to derive the ion concentrarions of higher ionization potential ions such as $\mathrm{O}^{+2}, \mathrm{Ar}^{+2}, \mathrm{Ar}^{+3}, \mathrm{Ne}^{+2}$, and the [NII] temperature for lower ionization potential ions like $\mathrm{O}^{+}, \mathrm{N}^{+}, \mathrm{S}^{+}$. In the cases when only one temperature was available, it was used for all ions. For the objects A 20 and NGC 1514 no temperatures were available, so that an average value of $10^{4} \mathrm{~K}$ was adopted. Concerning densities, for these nebulae the [SII] ratio was not available, and a typical value of $5000 \mathrm{~cm}^{-3}$ was adopted. Table 3 shows the adopted electron densities $\left(\mathrm{cm}^{-3}\right)$ and temperatures $(\mathrm{K})$ for all objects.

Ion abundances were calculated using a three-level atom model, by solving the statistical equilibrium equations, including radiative and collisional transitions. Elemental abundances were then derived using ionization correction factors to account for unobserved ions of each element. The atomic data and ICFs used in this work are the same as those adopted by Escudero \& Costa (2001), to which the reader is referred for details. The adopted formulae for the ICFs are given below:

$$
\begin{aligned}
& \frac{\mathrm{He}}{\mathrm{H}}=\frac{\mathrm{He}^{+}}{\mathrm{H}^{+}}+\frac{\mathrm{He}^{++}}{\mathrm{H}^{+}} \\
& \frac{\mathrm{O}}{\mathrm{H}}=\left(\frac{\mathrm{O}^{++}}{\mathrm{H}^{+}}+\frac{\mathrm{O}^{+}}{\mathrm{H}^{+}}\right) \frac{\mathrm{He}}{\mathrm{He}^{+}} \\
& \frac{\mathrm{N}}{\mathrm{H}}=\frac{\mathrm{N}^{+}}{\mathrm{H}^{+}} \frac{\mathrm{O}}{\mathrm{O}^{+}} \\
& \frac{\mathrm{S}}{\mathrm{H}}=\left(\frac{\mathrm{S}^{+}}{\mathrm{H}^{+}}+\frac{\mathrm{S}^{++}}{\mathrm{H}^{+}}\right)\left(1-\left(1-\frac{\mathrm{O}^{+}}{\mathrm{O}}\right)^{3}\right)^{-1 / 3} \\
& \frac{\mathrm{Ar}}{\mathrm{H}}=1.34 \frac{\mathrm{Ar}^{++}}{\mathrm{H}^{+}} \frac{\mathrm{O}}{\mathrm{O}^{++}} \\
& \frac{\mathrm{Ne}}{\mathrm{H}}=\frac{\mathrm{Ne}^{++}}{\mathrm{H}^{+}} \frac{\mathrm{O}}{\mathrm{O}^{++}} .
\end{aligned}
$$

For the cases where [SIII] 6312 is not available, we adopted the expression given by Kingsburgh \& Barlow (1994) to estimate the $\mathrm{S}^{++}$abundance. As in Escudero \& Costa (2001), we calculated helium abundances using the recombination theory, with recombination coefficients from Péquignot et al. (1991), and correcting the He I abundance for collisional effects using the correction terms from Kingdon \& Ferland (1995). He I abundances were determined as weighted averages from the 4471, 5876 and 6678 lines, using the line fluxes as weights. He II abundances were derived from the 4686 line. The obtained ion abundances are given in Table 4, where "E-05" means $10^{-05}$, etc.

\subsection{Abundances and distance-independent correlations}

The derived elemental abundances are given in Table 5. The helium abundance $(\mathrm{He} / \mathrm{H})$ is relative to hydrogen, and the remaining elemental abundances are given as $\epsilon(\mathrm{X})=\log (\mathrm{X} / \mathrm{H})+12$. The N/O ratio is also given, as this ratio is useful for classifying the nebulae, and is also related to the progenitor star mass. We have also examined the classification of the objects in our sample according to the classification scheme proposed by Peimbert (1978), which relies basically on elemental abundances. All nebulae in the sample are disk objects, belonging to types I-III. Subtypes IIa and IIb (Faúndez-Abans \& Maciel 1987) have also been included. Bulge (type V) or halo (type IV) objects were avoided (see the last column of Table 5).

Average helium abundances in our sample are $\mathrm{He} / \mathrm{H}=0.160$ for type I $\mathrm{PN}$ and $\mathrm{He} / \mathrm{H}=0.107$ for type II objects. For oxygen, we obtain $\epsilon(\mathrm{O})=8.42$ and $\epsilon(\mathrm{O})=8.66$, respectively, which shows some indication of $\mathrm{ON}$ cycling in type I PN, presumably those with more massive progenitors in which the second dredge-up process has operated (see for example de Freitas Pacheco 1993; Kingsburgh \& Barlow 1994).

The relative abundances can be better examined using distance-independent correlations, as can be seen in Fig. 1, 
Table 4. Ionic abundances.

\begin{tabular}{|c|c|c|c|c|c|c|c|c|}
\hline Object & $\mathrm{He} \mathrm{I}$ & $\mathrm{He}$ II & N II & S II & S III & O II & O III & Ar III \\
\hline A 12 & 0.111 & 0.008 & $8.45 \mathrm{E}-05$ & $2.82 \mathrm{E}-06$ & $3.23 \mathrm{E}-06$ & $6.21 \mathrm{E}-04$ & $1.69 \mathrm{E}-04$ & $9.03 \mathrm{E}-07$ \\
\hline A 18 & 0.141 & 0.010 & $4.27 \mathrm{E}-05$ & $5.25 \mathrm{E}-07$ & $1.87 \mathrm{E}-06$ & $4.43 \mathrm{E}-05$ & $4.60 \mathrm{E}-05$ & $5.25 \mathrm{E}-07$ \\
\hline A 20 & 0.042 & 0.083 & & $1.59 \mathrm{E}-07$ & $7.68 \mathrm{E}-06$ & $4.63 \mathrm{E}-05$ & $1.66 \mathrm{E}-04$ & $9.93 \mathrm{E}-08$ \\
\hline H 3-75 & 0.069 & 0.003 & $2.50 \mathrm{E}-05$ & $9.15 \mathrm{E}-07$ & $2.15 \mathrm{E}-06$ & $1.45 \mathrm{E}-04$ & $1.77 \mathrm{E}-04$ & $6.68 \mathrm{E}-07$ \\
\hline IC 2003 & 0.039 & 0.054 & $1.50 \mathrm{E}-06$ & $7.99 \mathrm{E}-08$ & $1.25 \mathrm{E}-06$ & $1.07 \mathrm{E}-05$ & $2.04 \mathrm{E}-04$ & $4.63 \mathrm{E}-07$ \\
\hline IC 2165 & 0.047 & 0.029 & $2.51 \mathrm{E}-06$ & $9.38 \mathrm{E}-08$ & $1.75 \mathrm{E}-06$ & $9.28 \mathrm{E}-06$ & $2.09 \mathrm{E}-04$ & $4.98 \mathrm{E}-07$ \\
\hline J 320 & 0.101 & 0.006 & $2.25 \mathrm{E}-07$ & $2.33 \mathrm{E}-08$ & $9.80 \mathrm{E}-07$ & $4.23 \mathrm{E}-06$ & $2.57 \mathrm{E}-04$ & $4.06 \mathrm{E}-07$ \\
\hline J 900 & 0.056 & 0.038 & $4.22 \mathrm{E}-06$ & $1.44 \mathrm{E}-07$ & $1.33 \mathrm{E}-06$ & $2.96 \mathrm{E}-05$ & $2.47 \mathrm{E}-04$ & $4.75 \mathrm{E}-07$ \\
\hline K 1-7 & 0.100 & 0.016 & $2.79 \mathrm{E}-05$ & $1.07 \mathrm{E}-06$ & $1.11 \mathrm{E}-06$ & $1.16 \mathrm{E}-04$ & $8.60 \mathrm{E}-05$ & $5.33 \mathrm{E}-07$ \\
\hline K 2-1 & 0.056 & 0.066 & $1.89 \mathrm{E}-05$ & $3.25 \mathrm{E}-07$ & $2.41 \mathrm{E}-06$ & $1.63 \mathrm{E}-04$ & $2.52 \mathrm{E}-04$ & $1.02 \mathrm{E}-06$ \\
\hline K 3-66 & 0.083 & & $9.26 \mathrm{E}-06$ & $8.93 \mathrm{E}-08$ & $2.03 \mathrm{E}-06$ & $4.83 \mathrm{E}-05$ & $1.21 \mathrm{E}-04$ & $6.86 \mathrm{E}-07$ \\
\hline K 3-70 & 0.138 & 0.023 & $1.16 \mathrm{E}-04$ & $9.61 \mathrm{E}-07$ & $2.14 \mathrm{E}-06$ & $1.05 \mathrm{E}-04$ & $7.30 \mathrm{E}-05$ & $7.09 \mathrm{E}-07$ \\
\hline M 1-6 & 0.081 & & $2.42 \mathrm{E}-05$ & $1.91 \mathrm{E}-07$ & $1.73 \mathrm{E}-06$ & $1.88 \mathrm{E}-04$ & $3.12 \mathrm{E}-05$ & $4.34 \mathrm{E}-07$ \\
\hline M 1-7 & 0.132 & 0.005 & $5.97 \mathrm{E}-05$ & $9.79 \mathrm{E}-07$ & $5.58 \mathrm{E}-06$ & $2.26 \mathrm{E}-04$ & $3.85 \mathrm{E}-04$ & $3.45 \mathrm{E}-06$ \\
\hline M 1-8 & 0.122 & 0.034 & $1.02 \mathrm{E}-04$ & $1.20 \mathrm{E}-06$ & $1.27 \mathrm{E}-06$ & $2.56 \mathrm{E}-04$ & $1.28 \mathrm{E}-04$ & $1.10 \mathrm{E}-06$ \\
\hline M 1-9 & 0.062 & 0.001 & $1.12 \mathrm{E}-05$ & $1.36 \mathrm{E}-07$ & $1.07 \mathrm{E}-06$ & $8.93 \mathrm{E}-05$ & $1.18 \mathrm{E}-04$ & $4.17 \mathrm{E}-07$ \\
\hline M 1-13 & 0.122 & 0.012 & $1.32 \mathrm{E}-04$ & $1.23 \mathrm{E}-06$ & $5.56 \mathrm{E}-06$ & $4.80 \mathrm{E}-04$ & $7.79 \mathrm{E}-04$ & $4.74 \mathrm{E}-06$ \\
\hline M 1-14 & 0.086 & & $1.59 \mathrm{E}-05$ & $2.23 \mathrm{E}-07$ & $5.95 \mathrm{E}-07$ & $1.53 \mathrm{E}-04$ & $4.79 \mathrm{E}-05$ & $3.64 \mathrm{E}-07$ \\
\hline M 1-16 & 0.104 & 0.023 & $1.40 \mathrm{E}-04$ & $3.42 \mathrm{E}-07$ & $1.18 \mathrm{E}-06$ & $2.40 \mathrm{E}-04$ & $3.46 \mathrm{E}-04$ & $1.45 \mathrm{E}-06$ \\
\hline M 1-17 & 0.069 & 0.011 & $3.86 \mathrm{E}-05$ & $1.50 \mathrm{E}-06$ & $6.29 \mathrm{E}-06$ & $1.61 \mathrm{E}-04$ & $4.67 \mathrm{E}-04$ & $1.57 \mathrm{E}-06$ \\
\hline M 1-18 & 0.136 & 0.017 & $9.21 \mathrm{E}-05$ & $1.14 \mathrm{E}-06$ & $5.97 \mathrm{E}-06$ & $1.75 \mathrm{E}-04$ & $1.91 \mathrm{E}-04$ & $1.38 \mathrm{E}-06$ \\
\hline M 3-2 & 0.134 & 0.077 & $1.80 \mathrm{E}-04$ & $1.21 \mathrm{E}-06$ & $6.03 \mathrm{E}-07$ & $8.95 \mathrm{E}-05$ & $1.74 \mathrm{E}-05$ & $2.51 \mathrm{E}-07$ \\
\hline M 3-3 & 0.100 & 0.029 & $1.79 \mathrm{E}-04$ & $3.00 \mathrm{E}-07$ & $1.22 \mathrm{E}-04$ & $1.51 \mathrm{E}-04$ & $1.22 \mathrm{E}-04$ & $8.38 \mathrm{E}-07$ \\
\hline NGC 1514 & 0.076 & 0.005 & & $1.63 \mathrm{E}-07$ & $3.40 \mathrm{E}-06$ & $1.96 \mathrm{E}-04$ & $4.15 \mathrm{E}-04$ & $4.48 \mathrm{E}-07$ \\
\hline SA $2-21$ & 0.110 & 0.019 & $1.67 \mathrm{E}-05$ & $5.72 \mathrm{E}-07$ & $2.38 \mathrm{E}-06$ & $2.99 \mathrm{E}-05$ & $1.44 \mathrm{E}-04$ & $9.65 \mathrm{E}-07$ \\
\hline YC 2-5 & 0.017 & 0.054 & & & & & $2.66 \mathrm{E}-04$ & $1.74 \mathrm{E}-07$ \\
\hline
\end{tabular}

Table 5. Abundances.

\begin{tabular}{lcccccrr}
\hline \hline Object & $\mathrm{He} / \mathrm{H}$ & $\epsilon(\mathrm{O})$ & $\epsilon(\mathrm{N})$ & $\epsilon(\mathrm{S})$ & $\epsilon(\mathrm{Ar})$ & $\log (\mathrm{N} / \mathrm{O})$ & Type \\
\hline A 12 & 0.119 & 8.93 & 8.06 & 6.78 & 6.78 & -0.87 & IIa \\
A 18 & 0.152 & 7.99 & 7.97 & 6.40 & 6.17 & -0.02 & I \\
A 20 & 0.125 & 8.80 & & 6.66 & 6.86 & & IIa \\
H 3-75 & 0.071 & 8.52 & 7.76 & 6.52 & 6.23 & -0.76 & IIb \\
IC 2003 & 0.094 & 8.71 & 7.86 & 6.53 & 6.19 & -0.85 & IIb \\
IC 2165 & 0.076 & 8.55 & 7.98 & 6.64 & 6.05 & -0.57 & IIb \\
J 320 & 0.107 & 8.44 & 7.17 & 6.45 & 5.77 & -1.27 & IIb \\
J 900 & 0.094 & 8.67 & 7.82 & 6.42 & 6.08 & -0.85 & IIb \\
K 1-7 & 0.115 & 8.37 & 7.75 & 6.36 & 6.29 & -0.62 & IIb \\
K 2-1 & 0.122 & 9.19 & 8.26 & 6.62 & 6.28 & -0.93 & IIa \\
K 3-66 & 0.083 & 8.23 & 7.51 & 6.39 & 6.11 & -0.72 & IIb \\
K 3-70 & 0.161 & 8.32 & 8.36 & 6.51 & 6.43 & 0.04 & I \\
M 1-6 & 0.081 & 8.34 & 7.45 & 6.28 & 6.61 & -0.89 & IIb \\
M 1-7 & 0.137 & 8.80 & 8.23 & 6.86 & 6.88 & -0.57 & IIa \\
M 1-8 & 0.156 & 8.69 & 8.29 & 6.41 & 6.75 & -0.40 & IIa \\
M 1-9 & 0.063 & 8.32 & 7.42 & 6.11 & 6.00 & -0.90 & IIb \\
M 1-13 & 0.134 & 9.14 & 8.58 & 6.88 & 7.05 & -0.56 & IIa \\
M 1-14 & 0.086 & 8.30 & 7.32 & 5.91 & 6.31 & -0.98 & III \\
M 1-16 & 0.127 & 8.86 & 8.62 & 6.23 & 6.60 & -0.24 & IIa \\
M 1-17 & 0.081 & 8.86 & 8.24 & 6.98 & 6.52 & -0.62 & IIb \\
M 1-18 & 0.153 & 8.62 & 8.34 & 6.88 & 6.60 & -0.28 & I \\
M 3-2 & 0.212 & 8.23 & 8.53 & 6.27 & 6.51 & 0.30 & I \\
M 3-3 & 0.129 & 8.54 & 8.62 & 6.49 & 6.51 & 0.08 & I \\
NGC 1514 & 0.081 & 8.81 & & 6.61 & 5.97 & & IIb \\
SA 2-21 & 0.129 & 8.31 & 8.05 & 6.61 & 6.26 & -0.22 & IIa \\
YC 2-5 & 0.071 & 9.05 & & & 6.00 & & IIb \\
\hline
\end{tabular}




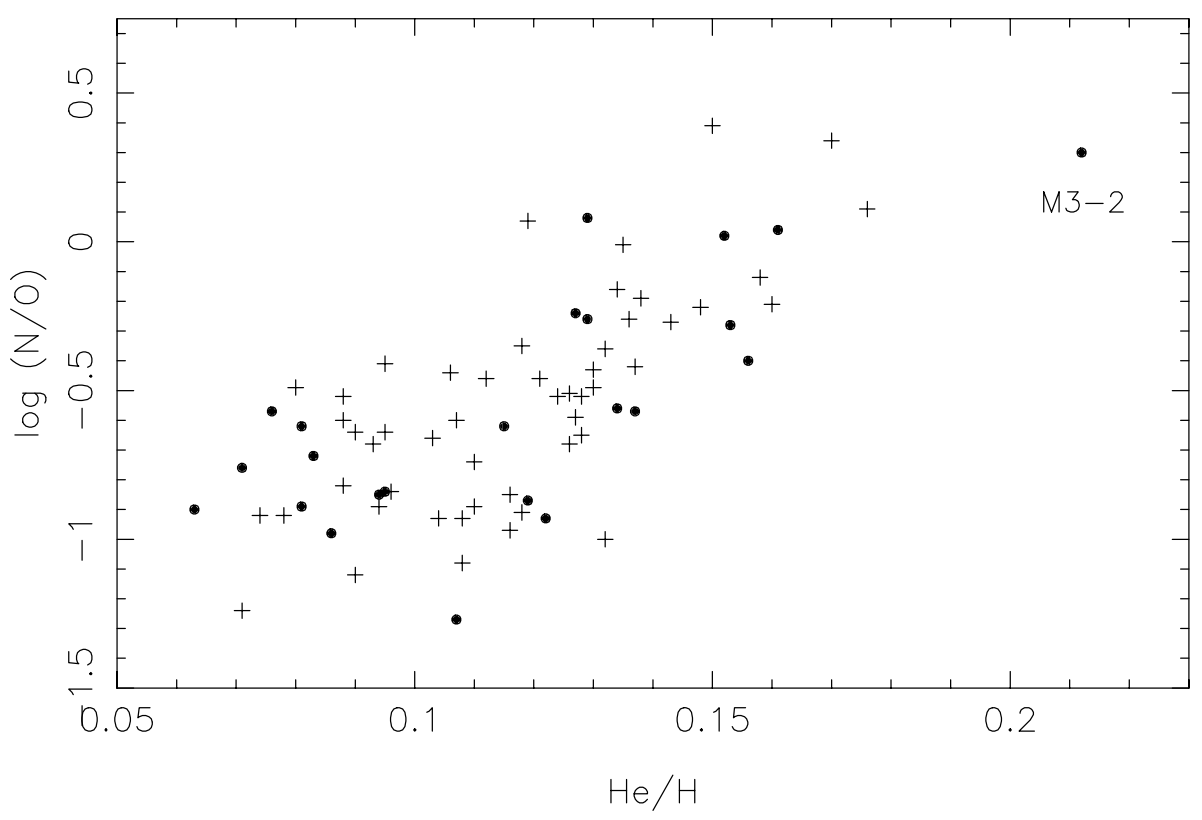

Fig. 1. $\log (\mathrm{N} / \mathrm{O})$ as a function of the helium abundance. Our new results are presented as solid dots, while the crosses show the abundances derived in our previous work, as quoted in the text.

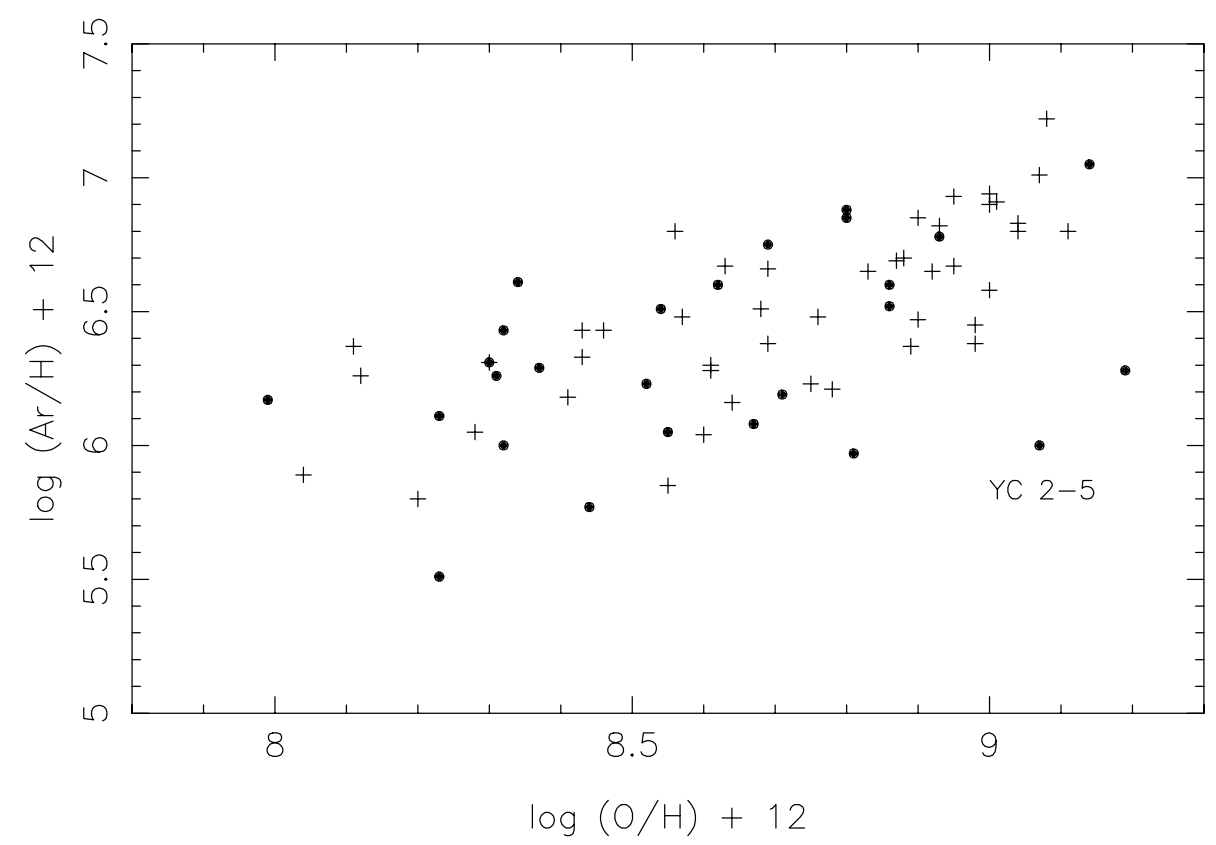

Fig. 2. Argon abundances as a function of the $\mathrm{O} / \mathrm{H}$ ratio. The symbols are as in Fig. 1.

which shows $\log (\mathrm{N} / \mathrm{O}) \times \mathrm{He} / \mathrm{H}$. Our new data are presented as solid dots, and the crosses show the results of our previous work as given by Costa et al. (1996), de Freitas Pacheco et al. (1989, 1991, 1992), and Maciel et al. (1990). As can be seen in the previous papers, all objects have been observed and analyzed in a homogeneous way, so that the total sample includes about 80 nebulae in the disk alone, forming one of the largest databases of accurate abundances of planetary nebulae available. This is important, since distance-independent correlations as shown by Fig. 1 are much better defined when a large sample is considered. It can be seen that our new data fit rather nicely the observed correlation.
The plot shows the expected trend of an increase in N/O as $\mathrm{He} / \mathrm{H}$ increases, reflecting the enrichment of $\mathrm{He}$ and $\mathrm{N}$ due to dredge-up episodes, as predicted by stellar evolution models. Some N/O enrichment can also be observed by comparing the data in Fig. 1 (or Table 5) with the corresponding values of the N/O ratio for HII regions of similar metallicity, measured by the $\mathrm{O} / \mathrm{H}$ ratio, as given for example by Henry \& Worthey (1999).

Figures 2 and 3 show the correlations with the $\mathrm{O} / \mathrm{H}$ ratio of the $\mathrm{Ar} / \mathrm{H}$ and $\mathrm{S} / \mathrm{H}$ abundances, respectively. These correlations are also well defined, and reflect the mass distribution of PN progenitors, as younger, more massive objects are expected 


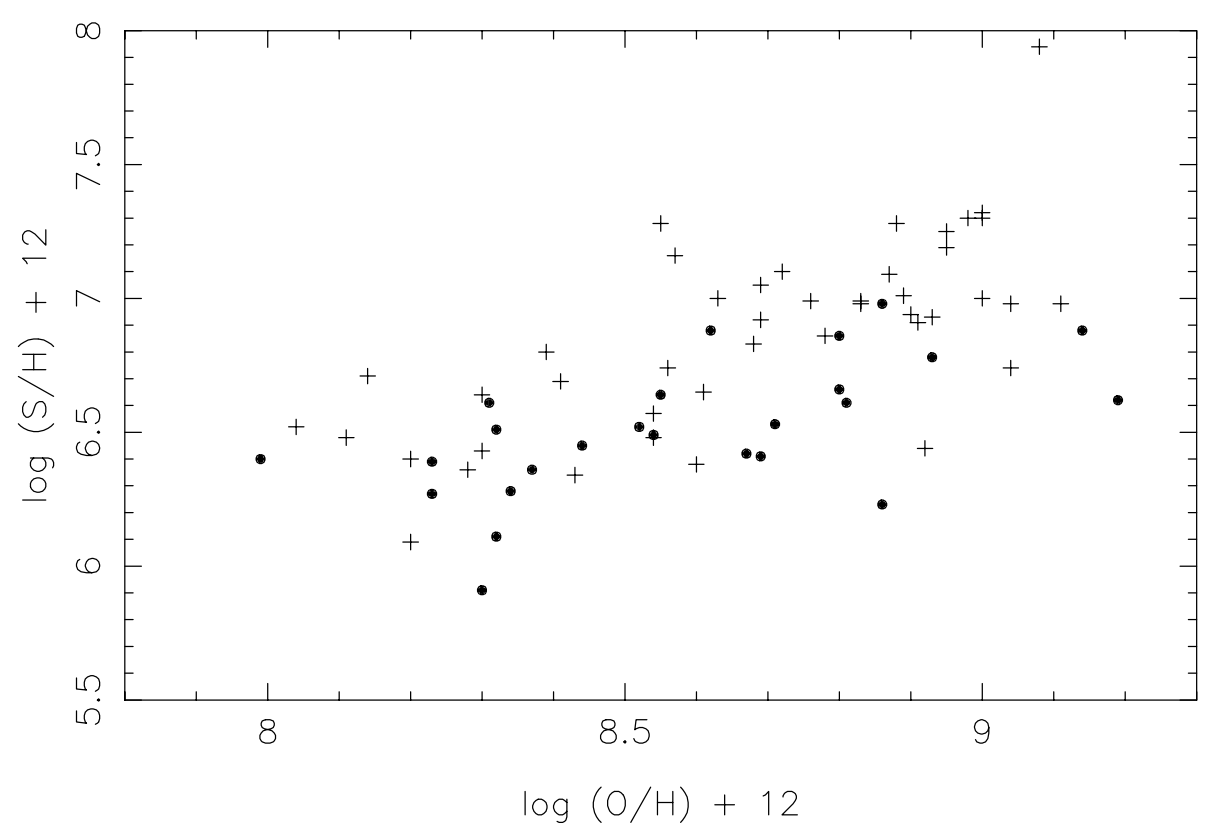

Fig. 3. Sulfur abundances as a function of the $\mathrm{O} / \mathrm{H}$ ratio. The symbols are as in Fig. 1.

to form from an interstellar medium richer in alpha-elements. The Ar abundance of the nebula YC 2-5 (Sa 2-22) shows a larger deviation from the trend than the remaining objects, as indicated in Fig. 2. This object shows a normal He abundance, and its oxygen abundance can also be considered normal, albeit larger than the average. If that is true, the argon abundance is probably a lower limit, which may be due to the fact that its electron density is not accurately determined.

\subsection{Comparison with previous results from the literature}

Most of the nebulae studied in this work are objects located at relatively large distances from the sun, for which accurate elemental abundances are being determined for the first time. Some objects, however, have been studied by other groups, so that it is interesting to compare our new results with previously determined abundances in the literature.

Considering first the earlier work, the objects IC 2003, IC 2165, J320 and J900 are part of the detailed study by Aller \& Czyzak (1983) based on image-tube scanner measurements and making use of theoretical models to represent individual line intensities. Regarding the $\mathrm{He} / \mathrm{H}$ abundances, our data agree with their results within 4\% for IC 2003, J320 and J900, with a larger discrepancy of about $38 \%$ for IC 2165 , for which the abundance by Aller \& Czyzak (1983) is higher than ours. Our electron temperatures for this object do not differ from the adopted values by Aller \& Czyzak (1983) and more recently by Kholtygin (1998) by more than $8 \%$. However, a larger discrepancy in the electron temperatures of 11 to $16 \%$ and in the helium abundance again of $38 \%$ is apparent for this nebula from the detailed work by Hyung (1994), so that our ionization correction factor may not account completely for the total abundance, and our result is probably underestimated. Considering now the heavy elements O, N, S and Ar, for IC 2003, IC 2165 and J900 the agreement is good, with an average discrepancy of a factor 1.3. The worst case is the Ar abundance of IC 2165, where the discrepancy reaches about a factor 1.8 , which is probably a result of the weaker lines of this element, or may be due the adopted electron temperature. For these elements the difference of our results to the analysis by Hyung (1994) is by a factor 1.4 on average. Note however that our oxygen abundance agrees very well with the more recent work of Kholtygin (1998), as we will see later on in this section. For J320 the agreement is very good for oxygen, which is well measured in both works, and poorer for the other elements. It should be noted that the sulfur and nitrogen abundances in the work of Aller \& Czyzak (1983) are of quality C or D, and that some recent work that we will quote later also produces discrepant results, which may be an indication of some variation inside the nebula.

In the spectrophotometric surveys performed by Köppen et al. (1991) and Cuisinier et al. (1996), about 140 planetary nebulae were studied on the basis of good quality spectra in which plasma diagnostics were tested with theoretical photoionization models. The first survey has four objects in common with our work, namely J320, J900, M 1-6 and M 3-2. $\mathrm{The} \mathrm{He} / \mathrm{H}$ abundances for these objects are in agreement with our results within an average of $26 \%$. The worst cases are J320 and M 1-6. In the former, the helium abundance was probably underestimated by Köppen et al. (1991), as our value agrees very well with that of Aller \& Czyzak (1983) and with the recent work of Milingo et al. (2002), as we will see later on in this section. For M 1-6, the helium abundance obtained by Köppen et al. (1991) is reportedly less accurate, due to incompleteness of the ionization correction. Regarding the heavy elements, the average discrepancy between our results and those by Köppen et al. (1991) is a factor 1.3, which means excellent agreement, for all elements and objects well measured by them, that is, those not marked with a colon (:). The remaining elements, 
for which their abundances are reportedly less accurate, present a much larger deviation, so that we believe our measurements do represent an improvement in the knowledge of these objects.

In the second study by Cuisinier et al. (1996), featuring nebulae located above the galactic plane, chemical abundances were derived for the objects M 1-9, M 1-16 and YC 2-5. Considering the first two, the $\mathrm{He} / \mathrm{H}$ abundances agree with our values within $20 \%$, and the heavy elements show an average discrepancy of a factor 1.8, which is especially large for argon. For YC 2-5 the discrepancy is higher, about a factor 2.5 , but it should be mentioned that Cuisinier et al. (1996) could not derive the sulfur abundance, and their argon abundance is uncertain due to incompleteness of the ionization correction. On the other hand, we could not measure the nitrogen abundance, which is indeed very low according to Cuisinier et al. (1996). Also, its odd position in Fig. 2 could be conciliated if its oxygen abundance is lower, as suggested by Cuisinier et al. (1996). Clearly, this object deserves further study.

The comprehensive work of Kingsburgh \& Barlow (1994, hereafter KB94) is based on optical spectrophotometric observations and UV data from the IUE satellite. Five nebulae from Table 1 (IC 2003, J900, M 1-8, M 1-13, and M 3-3) are included in the KS94 sample, for which a generally good agreement is observed in the abundances within the uncertainties of each work. This is particularly interesting, as the abundances derived by KB94 use independent ICFs, which are based on detailed photoionization models. For the $\mathrm{He} / \mathrm{H}$ ratio, the abundances of these objects derived by KS94 show an average deviation of $\Delta(\mathrm{He} / \mathrm{H})=0.018$ relative to our data, which corresponds roughly to $15 \%$. The worst case is J900, for which a discrepancy of about $30 \%$ is obtained, but, as we will see later, our value is in very good agreement with the recent result by Kwitter et al. (2003). For the heavy elements, the average deviations (dex) of the KS94 sample relative to our data are $\Delta \epsilon(\mathrm{O})=0.14, \Delta \epsilon(\mathrm{N})=0.15, \Delta \epsilon(\mathrm{Ar})=0.27$, and $\Delta \epsilon(\mathrm{S})=0.32$, which reflects the fact that the sulfur and argon abundances are generally less well known than the oxygen and nitrogen data, in view of their lower relative abundances. The average discrepancy factors for oxygen and nitrogen are about 1.5 , which is quite reasonable. For argon the discrepancy factor is somewhat larger, roughly 2.2. For sulfur we get a factor of 1.4 for the objects IC 2003, M 1-8 and M 1-13. For J 900 and M 3-3 the agreement of the results by KB94 and our data is poor, but the sulfur abundances of these objects were not well determined in the work of KB94.

Perinotto \& Corradi (1998) analyzed the chemical structure of some bipolar planetary nebulae by means of long slit spectrophotometry, considering several regions inside the nebulae in order to detect spatial abundance variations through the nebulae. There are three objects in common with our work, namely M 1-13, M 1-16 and M 3-2. The He/H abundances for these objects are similar to our values within $20 \%$, especially the relatively large abundance found in M 3-2, which is close to our result and also to the data of Köppen et al. (1991), showing that this object is really helium-rich, as can be seen in Fig. 1. The abundances of the heavy elements $\mathrm{O}, \mathrm{N}, \mathrm{S}$ and Ar are in agreement with our values within a factor of 2 , on average, with a better agreement for M 1-16 and M 3-2 and a poorer one for M 1-13, for which our results are closer to those by KB94.

Kholtygin (1998) determined oxygen abundances of about 70 planetary nebulae taking into account the effect of small temperature and density fluctuations on the line intensities of the nebular lines. The objects IC 2003, IC 2165, J320 and J900 are included in his sample, for which the oxygen abundances are $\epsilon(\mathrm{O})=8.70,8.50,8.58$ and 8.45, respectively. The agreeement with our results is excellent, within $10 \%$ for the first three nebulae and by a factor 1.7 for J900. The case of IC 2165 is particularly interesting, as we have mentioned the discrepancy of the helium abundance and electron temperature with some previous work.

The objects J320 and J900 were part of a recent survey of $\mathrm{PN}$ which aimed at providing a homogeneous spectroscopic database in the wavelength range of 3600-9600 $\AA$ (Milingo et al. 2002; Kwitter et al. 2003). This survey had the advantage of using the near infrared lines of [SIII] $\lambda$ 9069, $9532 \AA$, so that their abundances are expected to be more accurate, especially for sulfur, due to a better determination of the ion abundances of $\mathrm{S}^{+2}$. A comparison of our results for $\mathrm{J} 900$ (Kwitter et al. 2003) shows a very good agreement for all elements of better than 0.2 dex, or with an average discrepancy of a factor 1.3. This supports our data for this nebula in view of the discrepancies found relative to some of the earlier work already quoted, especially for nitrogen (Aller et al. 1983), argon (Köppen et al. 1991), oxygen (Kholtygin 1998), and the elements argon and sulfur (Kingsburgh \& Barlow 1994). For J320 (Milingo et al. 2002) the agreement is good for oxygen and argon, with an average discrepancy of a factor 1.3 , that is, the abundance differences are within 0.2 dex. For sulfur, we find an abundance higher by a factor 2 , or $\Delta \epsilon \simeq 0.30 \mathrm{dex}$. In fact, both our results for the sulfur abundances of J320 and J900 confirm the remarks by Kwitter et al. (2003) and Milingo et al. (2002), in the sense that use of the optical lines only probably leads to an overestimate of the sulfur abundance. For nitrogen, there is no agreement as this element is probably underestimated in our sample.

\section{The radial abundance gradient}

One of the most interesting consequences of the determination of abundances of PN located in the galactic anticenter is the effect they might have on the determination of the space variations in the radial abundance gradients. An analysis by Maciel \& Quireza (1999, hereafter MQ99) suggested that the gradients probably flatten out at galactocentric distances larger than $R \simeq 10 \mathrm{kpc}$ (assuming $R_{0}=7.6$ for the galactocentric distance of the LSR), especially for the $\mathrm{O} / \mathrm{H}$ and $\mathrm{Ne} / \mathrm{H}$ ratios. It is therefore interesting to investigate whether the presently derived abundances follow the same trend.

We have thus produced a merged sample including the objects of MQ99 and the present results. This sample forms a homogeneous group, as the abundances in MQ99 were obtained using basically the same methods as here. In fact, all abundances are generally similar within the average uncertainty of about 0.1 to 0.2 dex. 


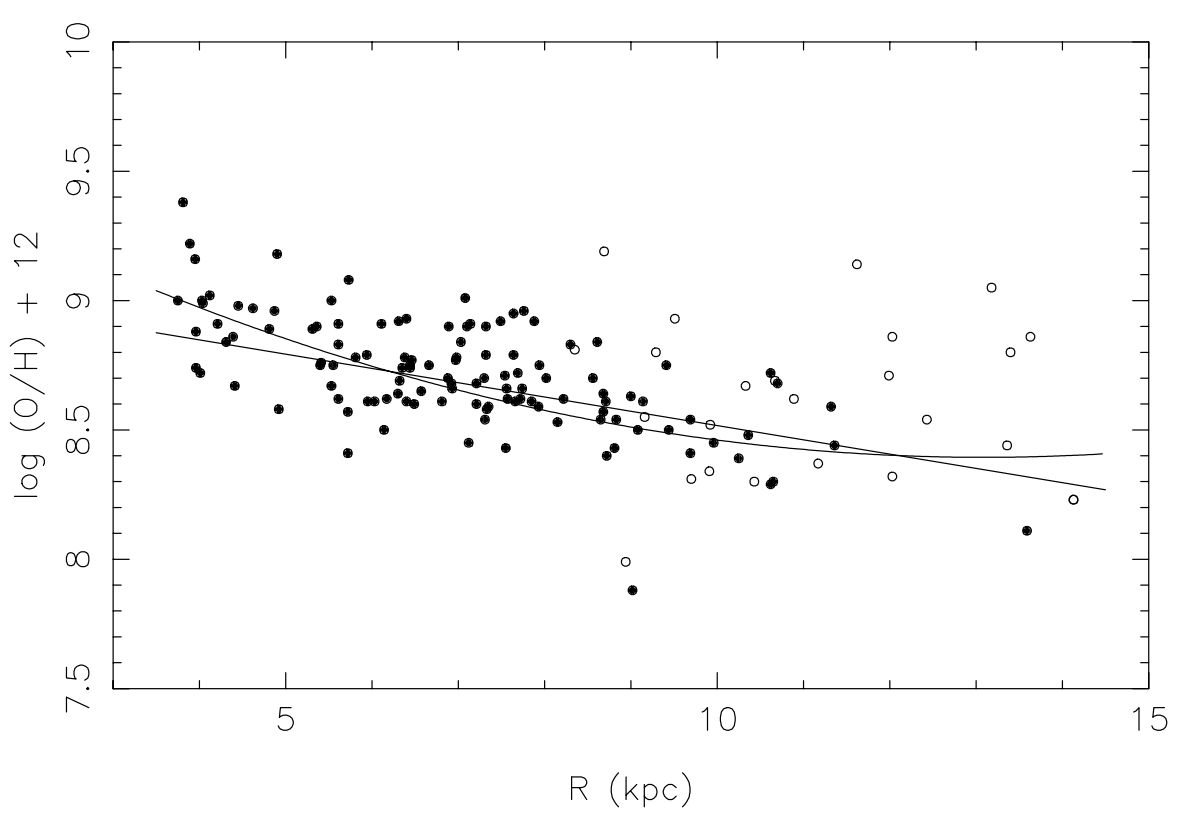

Fig. 4. The O/H gradient from the merged sample of Maciel \& Quireza (1999, filled circles) and the present sample (open circles). Linear and quadratic least squares fits are also shown.

In Fig. 4 we plot our new results for oxygen (open circles) along with the nebulae presented by MQ99 (filled circles). We have not included the object K3-70, for which apparently no reliable distance has been determined. We adopted the same galactocentric distance of the LSR as MQ99 and the PN distances are from Cahn et al. (1992), except for H3-75, for which we have used the distance given by Amnuel et al. (1984). The adopted heliocentric distances $d$ and the galactocentric distances $R(\mathrm{kpc})$ are shown in the last two columns of Table 1. Use of a different distance scale such as the catalogue by Maciel (1984) produces essentially the same results. As has been shown before (see for example Maciel \& Köppen 1994, hereafter MK94), the use of different statistical distances does not significantly affect the magnitudes of the gradients.

The results indicate that the radial abundance gradient, which is reasonably constant between galactocentric distances from 4 to $10 \mathrm{kpc}$, decreases for greater distances. The effect of the anticenter nebulae of the present work in the determination of the gradient is clearly seen in Fig. 4, as our sample was chosen specifically to cover galactocentric distances greater than $R_{0}$, so they compensate the lack of data for these distances in previous samples, such as MQ99 and MK94. This can also be seen by the least squares fits shown, both for a linear correlation (the straight line in Fig. 4) and a quadratic regression (curve). For the latter, the inner gradient is about $-0.09 \mathrm{dex} / \mathrm{kpc}$ for galactocentric radii between 4 and $5 \mathrm{kpc}$, reaching an essentially flat gradient for $R \geq 11 \mathrm{kpc}$. The average slope as indicated by the straight line is $-0.05 \mathrm{dex} / \mathrm{kpc}$, which is slightly lower than previous determinations for galactocentric distances close to $R_{0}$. The flattening at large distances is seen by the difference between the straight line and the parabola, especially at $R<5 \mathrm{kpc}$ and $R>12 \mathrm{kpc}$. This result supports other observational results for HII regions (see for example Vílchez \& Esteban 1996) and some theoretical models for the evolution of spiral galaxies (see Pagel 1997, and references therein).
It should be noted that the dispersion of the abundances increases at large galactocentric distances, probably due to the fact that the nebulae are located farther away than solar neighbourhood objetcs, which are better studied. For the remaining elements similar behaviour is observed, which is also seen in comparison with the earlier sample of Maciel \& Köppen (1994). For a detailed discussion of these effects the reader is referred to the recent work by Maciel \& Costa (2003) and Maciel et al. (2003).

It is interesting to compare the variation of the $\mathrm{O} / \mathrm{H}$ gradient with galactocentric radius as indicated by Fig. 4 with some recent results derived from different galactic objects. Data from O, B stars as discussed by Smartt (2000; see also Rolleston et al. 2000) suggest a constant slope for all galactocentric radii. The different behaviour of the two samples is probably related to the time variation of the $\mathrm{O} / \mathrm{H}$ gradient, as recently discussed by Maciel et al. (2003). According to that work, the average gradient, which can be roughly defined as the gradient from galactocentric radii of $4 \mathrm{kpc}$ to about $10 \mathrm{kpc}$, has flattened out from $-0.08 \mathrm{dex} / \mathrm{kpc}$ to about $-0.06 \mathrm{dex} / \mathrm{kpc}$ in the last $5 \mathrm{Gyr}$, allowing for an average uncertainty of about $0.2 \mathrm{dex} / \mathrm{kpc}$. Therefore, it is tempting to conclude that at the time when the oldest disk PN have been formed - several Gyr in the past the average gradient was somewhat steeper than indicated by younger objects such as O, B stars and HII regions. However, some flattening was already present near the outer edge of the Galaxy, probably reflecting the lower star formation rate in that region compared with the regions closer to the galactic center. Such flattening is apparent from our PN data, as can be seen in Fig. 4. As the galactic evolution proceeded, the flattening initially located near the outer Galaxy spread towards the more central regions, which explains the lower and approximately constant gradient shown by the O, B stars. Since the temporal flattening rate has decreased in the last few Gyr, as suggested by the results of Maciel et al. (2003), the gradients presented 
by young objects such as O, B stars and HII regions are expected to be similar, which is supported by the observations, again within an average uncertainty of $0.02 \mathrm{dex} / \mathrm{kpc}$. Such a scenario is also supported by recent work on HII regions, for which gradients as low as about $-0.04 \mathrm{dex} / \mathrm{kpc}$ have been proposed (Deharveng et al. 2000). On the theoretical side, gradients that flatten out in time with a decreasing flattening rate in the last few Gyr are supported by the recent models proposed by Hou et al. (2000).

Acknowledgements. This work was partly supported by the brazilian agencies FAPESP and CNPq. M.M.M.U. acknowledges FAPESP for her undergraduate fellowship (Process 00/02813-9). Observations at ESO/Chile were possible through the FAPESP grant 98/10138-8.

\section{References}

Acker, A., Ochsenbein, F., Stenholm, B., et al. 1992, ESO-Strasbourg catalogue of galactic planetary nebulae (Munich: ESO)

Aller, L. H., \& Czyzak, S. J. 1983, ApJS, 51, 211

Amnuel, P. R., Guseinov, O. H., Novruzova, H. I., \& Rustamov, Yu. S. 1984, Ap\&SS, 107, 19

Andrievsky, S. M., Kovtyukh, V. V., Luck, R. E., et al. 2002, A\&A, 392, 491

Blair, W. P., Kirshner, R. P., \& Chevalier, R. A. 1982, ApJ, 254, 50

Cahn, J. H., Kaler, J. B., \& Stanghellini, L. 1992, A\&AS, 94, 399

Cardelli, J. A., Clayton, G. C., \& Mathis, J. S. 1989, ApJ, 345, 245

Chiappini, C., Matteucci, F., \& Gratton, R. 1997, ApJ, 477, 765

Costa, R. D. D., Chiappini, C., Maciel, W. J., \& de Freitas Pacheco, J. A. 1996, A\&AS, 116, 249

Costa, R. D. D., Chiappini, C., Maciel, W. J., \& de Freitas Pacheco, J. A. 1997, in Advances in stellar evolution, ed. R. T. Rood, \& A. Renzini (Cambridge: CUP), 159

Cuisinier, F., Acker, A., \& Köppen, J. 1996, A\&A, 307, 215

Deharveng, L., Peña, M., Caplan, J., \& Costero, R. 2000, MNRAS, 311,329

Díaz, A. I. 1989, in Evolutionary phenomena in galaxies, ed. J. E. Beckman, \& B. E. J. Pagel (Cambridge: CUP), 377

Escudero, A. V., \& Costa, R. D. D. 2001, A\&A, 380, 300

Esteban, C., \& Peimbert, M. 1995, Rev. Mex. Astron. Astrofis. SC, 3 , 133

Faúndez-Abans, M., \& Maciel, W. J. 1987, A\&A, 183, 324

Ferguson, A. M. N., Gallagher, J. S., \& Wyse, R. F. G. 1998, AJ, 116, 673

de Freitas Pacheco, J. A. 1993, ApJ, 403, 673

de Freitas Pacheco, J. A., Costa, R. D. D., Maciel, W. J., \& Codina-Landaberry, S. J. 1989, Ann. Acad. Bras. Ci., 61, 389 de Freitas Pacheco, J. A., Maciel, W. J., Costa, R. D. D., \& Barbuy, B. 1991, A\&A, 250, 159

de Freitas Pacheco, J. A., Maciel, W. J., \& Costa, R. D. D. 1992, A\&A, 261, 579

Henry, R. B. C., \& Howard, J. W. 1995, ApJ, 438, 170

Henry, R. B. C., \& Worthey, G. 1999, PASP, 111, 919

Hou, J. L., Prantzos, N., \& Boissier, S. 2000, A\&A, 362, 921

Hyung, S. 1994, ApJS, 90, 119

Kaufer, A., Szeifert, Th., Krenzin, R., Baschek, B., \& Wolf, B. 1994, A\&A, 289, 740

Kennicutt, R. C., \& Garnett, D. R. 1996, ApJ, 456, 504

Kholtygin, A. F. 1998, A\&A, 329, 691

Kingdon, J., \& Ferland, G. J. 1995, ApJ, 442, 714

Kingsburgh, R. L., \& Barlow, M. J. 1994, MNRAS, 271, 257

Köppen, J., Acker, A., \& Stenholm, B. 1991, A\&A, 248, 197

Kwitter, K. B., Henry, R. B. C., \& Milingo, J. B. 2003, PASP, 115, 80

Maciel, W. J. 1984, A\&AS, 55, 253

Maciel, W. J. 1997, in Planetary Nebulae, ed. H. J. Habing, \& H. J. G. L. M. Lamers (Dordrecht: Kluwer), IAU Symp., 180, 397

Maciel, W. J. 2000, in The evolution of the Milky Way, ed. F. Matteucci, \& F. Giovannelli (Dordrecht: Kluwer), 81

Maciel, W. J., \& Costa, R. D. D. 2003, in Planetary Nebulae, ed. S. Kwok, M. Dopita, \& R. Sutherland (San Francisco: ASP), Proc. IAU Symp., 209, 551

Maciel, W. J., \& Köppen, J. 1994, A\&A, 282, 436

Maciel, W. J., \& Quireza, C. 1999, A\&A, 345, 629

Maciel, W. J., de Freitas Pacheco, J. A., \& Codina-Landaberry, S. J. 1990, A\&A, 239, 301

Maciel, W. J., Costa, R. D. D., \& Uchida, M. M. M. 2003, A\&A, 397, 667

Matteucci, F. 2000, in The evolution of the Milky Way, ed. F. Matteucci, \& F. Giovannelli (Dordrecht: Kluwer), 3

Milingo, J. B., Henry, R. B. C., \& Kwitter, K. B. 2002, ApJS, 138, 285

Osterbrock, D. E. 1989, Astrophysics of gaseous nebulae and active galactic nuclei (Santa Cruz: University Science Books)

Pagel, B. E. J. 1997, Nucleosynthesis and chemical evolution of galaxies (Cambridge: CUP)

Peimbert, M. 1978, in Planetary Nebulae, ed. Y. Terzian (Dordrecht: Reidel), IAU Symp., 76, 215

Peimbert, M., \& Carigi, L. 1998, in ASP Conf. Ser., 147, ed. D. Friedli, M. G. Edmunds, C. Robert, \& L. Drissen, 88

Péquignot, D., Petitjean, P., \& Boisson, C. 1991, A\&A, 251, 680

Perinotto, M., \& Corradi, R. L. M. 1998, A\&A, 332, 721

Rolleston, W. R. J., Smartt, S. J., Dufton, P. L., \& Ryans, R. S. I. 2000, A\&A, 353, 537

Searle, L. 1971, ApJ, 168, 327

Smartt, S. J. 2000, in The evolution of the Milky Way, ed. F. Matteucci, \& F. Giovannelli (Dordrecht: Kluwer), 323

Smith, H. E. 1975, ApJ, 199, 591

Vílchez, J. M., \& Esteban, C. 1996, MNRAS, 280, 720 